Roma

\title{
IL GIUDEO-ITALIANO E I PROBLEMI DELLA SUA DEFINIZIONE: UN CAPITOLO DI STORIA DELLA LINGUISTICA $^{1}$
}

\section{INTRODUZIONE}

Lo scopo di questo articolo è di portare all'attenzione dei linguisti che lavorano sull'italiano un argomento poco noto perché è stato trattato per lo più, per le caratteristiche del suo materiale, al di fuori dell'italianistica: il cosiddetto giudeo-italiano. Il termine si riferisce alle varietà dialettali usate in una serie di documenti che sono stati oggetto di studio, con poche eccezioni, da parte di specialisti di ebraico. I testi hanno, aldilà del loro immediato valore come documenti della cultura ebraica italiana, anche un interesse linguistico: è questa appunto l'angolatura di questo lavoro, perché il tentativo di definire tali parlate all'interno delle varietà di italiano ha avuto varie soluzioni da parte di studiosi diversi, che costituiscono un itinerario teorico molto interessante. Si tratta di uno spezzone di storia della linguistica italiana e romanza in cui si ripercorre un itinerario simile a quello della definizione di italiano standard. Si tratta di un percorso che è parallelo all'evoluzione della linguistica stessa e che è stato fino a non molto tempo fa, come si cercherà di dimostrare, dominato in larga parte dalla visione delle varietà linguistiche come sistemi discreti, unitari ed omogenei, propria della descrizione linguistica fino alla messa a punto dei modelli macrosociolinguistici che hanno incorporato sistematicamente la variazione e il continuo linguistico. In particolare nel nostro caso l'immagine del giudeo-italiano risentiva della concezione di un'entità quanto mai elusiva, che ha dominato la linguistica italiana, quella dell'italiano standard.

Il termine giudeo-italiano copre tutta una serie di documenti linguistici molto eterogenei e si riferisce a sistemi linguistici non più in uso. Infatti in Italia oggigiorno non abbiamo differenze di comportamento linguistico tra ebrei e non ebrei che siano tali da farci parlare di giudeo-italiano o di dialetti giudeo-italiani, come di un sistema o di una serie di sistemi a sé stanti in uso ${ }^{2}$. Il materiale in giudeo-italiano è diviso in

1 Ringrazio il Centro di Cultura Ebraica della Comunità Ebraica di Roma e il Centro Bibliografico dell'Unione delle Comunità Ebraiche Italiane per il loro cortese aiuto nell'aiutarmi a reperire la bibliografia per questo articolo.

2 L'unica eccezione è Roma dove alcuni individui, generalmente residenti nell'antico ghetto, presentano ancora tratti diversi dalla parlata regionale locale, ma si tratta di un fenomeno di entità molto ridotta. 
due corpus distinti e molto diversi tra di loro che sono stati analizzati da studiosi di discipline diverse.

Un corpus è quello redatto in alcuni dei dialetti regionali italiani (non quindi, malgrado il nome, in sottosistemi dell'italiano nazionale standard) come erano parlati dagli ebrei in diverse città d'Italia. Alla fine del secolo scorso appaiono i primi interventi sulle parlate italiane come vengono usate dagli ebrei nelle diverse città d'Italia (Sacerdote 1893 e Modona 1893). All'inizio di questo secolo fu lanciata una proposta da Cammeo (1909) su un periodico ebraico, Il Vessillo Israelitico, di raccogliere con l'aiu to dei lettori tutte le voci che, nelle diverse città d'Italia dove ci fosse una comunità ebraica, venivano usate comunemente solo dagli ebrei e non avevano circolazione al di fuori della comunità. Questa iniziativa ebbe un certo seguito e Cammeo continuò a pubblicare voci raccolte con l'aiuto dei lettori dal 1909 al 1911: alla discussione partecipa anche Camerini (1909: 358-9 e 1909: 505-6). L'iniziativa attirò anche l'attenzione di un noto studioso di ebraismo, U. Cassuto, che contribuì con una breve descrizione del giudeo-fiorentino, e soprattutto risvegliò l'interesse per queste varietà linguistiche che stavano scomparendo, che s'inquadra perfettamente nel clima culturale della fine dell'Ottocento, epoca della nascita degli studi glottologici e dialettali, da una parte, e etnologici dall'altra. Nel 1926, questa volta su La Rassegna Mensile d'Israel, viene lanciato un altro appello di raccolta da Bachi, in chiave apertamente antropologica.

Nasce così un filone antiquario di raccolta e catalogazione, in larga parte frutto dell'entusiasmo di non specialisti, il cui interesse dura tuttora, con tentativi di fissare o ricostruire le varietà moribonde sotto forma di poesie, testi teatrali (alcuni tradizionali altri scritti per far rivivere la parlata, come tuttora a Roma) o liste di unità lessicali ed espressioni linguistiche, tipiche dei soli parlanti ebrei. Il materiale inviato alla Rassegna consegnato verrà conservato e consegnato poi a Terracini che lo studierà dal punto di vista linguistico. Terracini ricevette anche altro materiale raccolto da Giacomelli $^{3}$, un appassionato di ricerche dialettali che aveva lavorato a Roma, Pitigliano, Ferrara e in altri centri. Terracini pubblicherà il frutto delle raccolte di Giacomelli in un articolo del 1962. Tutto questo materiale è in qualche modo di tipo orale anche se trascritto ed è stato in seguito oggetto di studio da parte di vari studiosi tra cui glottologi e dialettologi, alcuni dei quali hanno aggiunto raccolte nuove: si vedano, tra gli altri, Modena Mayer e Massariello Merzagora (1973) per il giudeo-modenese, Colorni (1971) per il giudeo-mantovano, Massariello Merzagora (1977 e 1980) che produce una rassegna generale delle diverse parlate come ci sono giunte, Fortis e Zolli (1979) per il giudeo-veneziano, Sestieri Scazzocchio (1970) e Pavoncello (1978, 1986, 1988), Milano (1963b) per il giudeo-romano, Beccani $(1941,1942)$ e Fornaciari (1983) per il giudeo-livornese.

3 Terracini, che lo conobbe personalmente, dà una presentazione bio-bibliografica di Giacomelli (1962). 
Accanto alle raccolte di voci fiorisce anche il desiderio di fissare poesie tradizionali e produrne di nuove: Terracini stesso nel 1938 pubblica e commenta due composizioni in versi. Nasce una fiorente produzione con I sonetti giudaico-romaneschi di Del Monte del 1927 (apparsi per la prima volta nel 1908), seguiti da altre due raccolte nel 1932 e 1955; Bedarida pubblica Ebrei di Livorno. Tradizioni e gergo in 180 sonetti giudaico-livornesi del 1956, seguiti da altri contributi minori, come Colombo (1970) per Moncalvo e Stock (1970) per il triestino.

Per il teatro abbiamo vari contributi di Bedarida a partire dal 1924 in poi, sempre in giudeo-livornese e un'interessante versione un giudeo-fiorentino di un canovaccio tradizionale La Gnora Luna edito dai Bené Kedem, pseudonimo dei figli dello studioso di ebraismo U. Cassuto. Esistono canovacci ripresi in giudeo-romano a cui recentemente s'è affiancata una produzione nuova sulla stessa scia che viene rappresentata da una compagnia locale: questo materiale teatrale è stato raccolto in un volume curato da Fortis (1989).

Come per tutte le raccolte antiquarie, l'oralità di questo materiale è ovviamente piuttosto dubbia, così come lo è in parte l'attendibilità delle forme stesse, spesso ricordate vagamente da chi in realtà non le usa più. Soprattutto dal punto di vista sociolinguistico manca un riferimento di contesto alle forme studiate, che ci giungono attraverso liste raccolte pazientemente, ma non all'interno di interviste dall'uso vivo. Tuttavia, poiché ormai sono l'unica fonte che abbiamo, questi resti costituiscono un corpus divenuto ormai classico sull'argomento e insostituibile.

L'altro filone, invece, appartiene all'ambito scritto ed è stato l'oggetto di studio di filologi ed esegeti, più che di linguisti. Si tratta di un'ampia messe di testi scritti coi caratteri dell'alfabeto ebraico in un italiano come era parlato e compreso dagli ebrei, quindi con caratteristiche proprie rispetto alle equivalenti varietà parlate dai gentili. La parte maggiore di questi documenti è di tipo religioso ed essi si situano cronologicamente tra i secoli XI e il XVIII. Poiché gli ebrei in Italia avevano smesso già in epoca romana di usare l'ebraico come lingua parlata, si presentava il problema che chi non lo conosceva non poteva seguire i testi sacri e recitare le preghiere prescritte. Gli studiosi e, in molti casi, gli uomini delle comunità avevano almeno una conoscenza della lingua scritta, però le donne e i bambini avevano bisogno di una traduzione per poter pregare e seguire le funzioni in sinagoga. Abbiamo perciò traduzioni della Bibbia; glosse al testo biblico; glossari di termini ebraici tradotti in italiano; traduzioni di preghiere, rituali e formulari; prediche e composizioni poetiche religiose. Oltre al materiale religioso abbiamo anche documenti legali, sempre scritti in italiano ma in caratteri ebraici, e una produzione poetica non religiosa, ma la parte laica del materiale è più ridotta ${ }^{4}$.

$4 \quad$ Una prima descrizione dei documenti scritti si trova in Cassuto (1929). Cuomo (1981: 429-435) offre una presentazione tipologica del materiale sia scritto che sui dialetti parlati, Freedman (1972, Cap. I) dà una bibliografia ragionata su tutto il materiale, mentre Colorni (1971), Massariello Merzagora (1977) e Fortis e Zolli (1979) rendono conto delle raccolte antiquarie, poetiche e teatrali e Fortis (1989) in particolare delia produzione teatrale. 
Il termine giudeo-italiano appare per la prima volta in un articolo di U. Cassuto (1909) ed è ormai diventato canonico, ma si presta ad equivoci, in quanto può far pensare ad una varietà linguistica sovraregionale come l'italiano letterario ${ }^{5}$. Sia i testi scritti che le raccolte di voci dei dialetti parlati documentano invece delle parlate regionali così come venivano usate dagli ebrei che, per ragioni intrinseche alla loro condizione, hanno dato loro una impronta peculiare. Per evitare equivoci si propone di usare qui varietà o parlate giudeo-italiane.

\section{CENNI DI STORIA DEGLI EBREI ITALIANI}

Prima di poterci addentrare in una discussione sull' origine e sui rapporti reciproci delle varietà giudeo-italiane, è bene mettere in rilievo alcuni tratti della storia degli ebrei italiani ${ }^{6}$, che sono fondamentali per impostare correttamente il problema linguistico.

La storia degli ebrei italiani è essenzialmente una storia di movimenti migratori particolari $\mathrm{e}$, in generale, più frequenti che per il resto della popolazione e di segregazione. Gli insediamenti più antichi in Italia sono quello di Roma e quelli dell' Italia meridionale, dove le comunità ebraiche erano già numerose e fiorenti nell' alto medioevo.

Con l'espulsione dal Regno delle Due Sicilie decretata dai Re Spagnoli nel sec. $\mathrm{XV}$, abbiamo un vasto movimento migratorio che muove da tutto il Sud. Nelle comunità del Meridione tutti coloro che non si convertirono scelsero di emigrare e la maggior parte di essi si trasferì a Roma, da dove molti si spostarono ancora più a Nord.

A parte questo flusso migratorio dalla direzione e datazione chiare e definite, vi furono una miriade di spostamenti da una città all'altra della Penisola, dovuti alle alterne vicende a cui le comunità erano sottoposte. Le concessioni di permanenza e di lavoro fatte agli ebrei cambiavano continuamente nei diversi luoghi, a seconda delle pressioni esercitate dalla Chiesa, delle condizioni economiche e perciò del bisogno che i governanti avevano occasionalmente di sviluppare questo o quel commercio o artigianato (per esempio quello della seta) o del prestito di capitali e a causa dell'intolleranza religiosa creata a ondate dai predicatori ${ }^{7}$. $\grave{E}$ difficile riassumere quella che fu una continua e particolare instabilità geografica e sociale: la formulazione più chiara e utile per noi in questa sede è che gli ebrei furono sollecitati e spinti a molti e continui spostamenti da una città all'altra, da uno Stato all'altro, più di qualsiasi altro gruppo ed è questa mobilità, diversa e maggiore di quella dei cristiani, che produrrà delle commistioni di forme linguistiche infra e intraregionali del tutto particolari. Ba-

5 È stato suggerito da Gold (1980) di sostituire questo termine con Italkian : si veda la discussione in proposito in Cuomo (1982: 9).

6 Per una storia degli ebrei italiani si veda A. Milano, Storia degli Ebrei italiani, Milano 1963a.

7 Per un quadro esauriente delle cause anche antropologiche-sociali delle cacciate e della segregazione si veda Bonfil 1991. 
sta leggere la storia delle diverse comunità italiane per vedere come in quasi ogni caso si assista a un andirivieni di concessioni e restrizioni di soggiorno, chiamate e cacciate, che resero estremamente mobili gli abitanti della maggioranza delle comunità. Poche di esse furono stabili: come eccezioni abbiamo quella romana e quella livornese (che è però una comunità relativamente tarda, di epoca rinascimentale) che godettero, pur nelle alterne vicende, almeno di una stabilità geografica. Quest'ultima non significò però stabilità linguistica perché le comunità stabili funsero da rifugio per gli ebrei in fuga dalle altre, anche straniere, francesi, tedesche, spagnole, portoghesi e quindi ricevettero apporti linguistici diversi.

A parte gli spostamenti geografici particolari e particolarmente numerosi, l'altra caratteristica delle comunità ebraiche fu la segregazione dal resto della popolazione. Già melle città medievali e nell'antichità, la popolazione ebraica conduceva una vita a parte, ma non si trattava necessariamente di una segregazione forzata. Nel migliore dei casi era quel fenomeno naturale per cui gruppi di immigrati tendono sempre a radunarsi nella stessa area di una città semplicemente in quanto condividono lo stesso stile di vita, gli stessi usi e costumi. Anche nelle migliori condizioni era naturale che la popolazione ebraica si radunasse insieme per facilitare il culto religioso e l'osservanza delle regole dietetiche. Spesso, però, era anche il rifiuto della popolazione cristiana e la conseguente autodifesa che costituivano una spinta alla separazione spontanea che veniva anche in parte incoraggiata dalle autorità.

A partire dalla prima metà del secolo XVI vengono istituiti i veri e propri ghetti, chiusi fisicamente da porte invalicabili di notte: a Venezia prima, nel 1516, e poi a Roma nel 1555. Su pressione dei Papi la segregazione coatta nei ghetti venne imposta con l'andar del tempo, in epoche diverse, anche negli altri Stati italiani dove vi fosse una comunità. L'unica eccezione rimane Livorno dove il ghetto non fu mai istituito. Agli effetti linguistici l'istituzione dei ghetti chiusi non fece che esasperare una coesione linguistica già presente all'interno delle comunità, dove si viveva spesso già nella cosiddetta "giudecca", il quartiere ebraico, che ancora esiste nella toponomastica di tanti paesi e città italiane.

\section{CARATTERISTICHE LINGUISTICHE DELLE VARIETÀ GIUDEO-ITALIANE ${ }^{8}$}

Le vicissitudini storiche delle Comunità ebraiche hanno dato alle varietà parlate al loro interno delle caratteristiche linguistiche particolari, che potremmo riassumere come: arcaicità, mistilinguismo italiano e mistilinguismo esterno all'italiano.

$8 \quad$ L'esistenza di una forma peculiare di lingua parlata e scritta dagli ebrei, con caratteristiche proprie rispetto a quella dei gentili, è un fenomeno che si trova in tutti i paesi in cui vi è stata una presenza ebraica. Può trattarsi di un sistema linguistico completamente diverso come lo yiddish in Germania e Europa orientale o il ladino (antico spagnolo) parlato ancora in Turchia e in Bosnia, o di una variante solo parzialmente diversa. L'interesse per le varianti linguistiche usate dagli ebrei nasce nel secondo Ottocento. Per quanto riguarda l'area romanza il primo ad avviare gli studi sulle cosiddette parlate 


\subsection{ARCAICITÀ}

La patina arcaica di queste parlate è la conseguenza del conservatorismo linguistico che si verifica sempre quando un gruppo di parlanti subisce una segregazione fisica e/o sociale. Le parlate dei quartieri ebraici prima e ancor più quelle dei ghetti in seguito, furono sottratte in parte all'evoluzione storica subita dalle parlate dei non ebrei che li circondavano. Non va dimenticato anche però che d'altra parte si verificavano delle innovazioni linguistiche all'interno delle comunità ed esse non venivano diffuse all'esterno, rimanendo pertanto forme caratteristiche solamente del gruppo: per esempio (Massariello 1977: 76-77) il giudeo-modenese mostrava di essere più italianizzante, e quindi innovativo, del dialetto non ebraico, data la preponderanza di borghesi all'interno della comunità.

In generale, però, in tutta la letteratura sull'argomento, per tutte le parlate scritte o orali di cui ci è giunta documentazione, appare una patina arcaica, che è stata accettata da tutti gli studiosi come una caratteristica fondamentale delle parlate giudeo-italiane. Essa d'altra parte è un fenomeno noto ai linguisti storici perché appare in molte altre situazioni dove si è avuta una segregazione di natura geografica, come per esempio in comunità linguistiche isolate in villaggi mal collegati col contesto geografico circostante.

Tuttavia, è stata attirata giustamente l'attenzione sul fatto che può accadere di attribuire al conservatorismo dei tratti linguistici arcaicizzanti la cui presenza può essere spiegata in altro modo: essi possono provenire da altri luoghi dove la parlata era più conservatrice anche per i non ebrei (differenza città-campagna) o essere dei casi di italianizzazione. L'arcaicità, inoltre, come la presenza di forme ebraiche, se da una parte è sempre presente, dall'altra incide quantitativamente in modo molto marcato solo laddove la segregazione è molto forte e può perciò essere presa come un indice delle condizioni sociali delle comunità.

\subsection{MISTILINGUISMO INTERNO}

L'altra caratteristica saliente è il mistilinguismo, cioè la presenza di forme provenienti da altre lingue o altre varietà, portate dal di fuori come conseguenza dei movimenti migratori particolari di cui s'è detto. Abbiamo due tipi di mistilinguismo: interno all'italiano ed esterno all'italiano.

giudeo-romanze fu Blondheim nel 1923, con la pubblicazione di uno studio comparativo dei termini romanzi usati per le traduzioni bibliche, avanzando in un suo ulteriore studio del 1925 l'ipotesi dell'esistenza di una vera e propria koiné romanza nella parte sud-occidentale dell'Europa. L'ipotesi di Blondheim ha avuto naturalmente una forte influenza sulla concezione che vedremo di una koiné ebraico-italiana unitaria (M. Weinreich 1956, Birnbaum 1942 e 1971 danno una visione del panorama globale delle varietà ebraiche; per l'origine delle varietà ashkenazite si veda Agus 1974). 
Il mistilinguismo interno a sua volta si divide in infraregionale, quando vengono importate forme dalle zone circostanti della stessa regione, e interregionale, quando $i$ prestiti provengono dal di fuori della regione, da dialetti diversi.

Le forme infraregionali giungevano nelle città con l'arrivo di gente da paesi e cittadine della provincia circostante, soprattutto in età recente con la mobilità creata dalla scomparsa dei ghetti e la conseguente urbanizzazione, che introduceva nei dialetti di città tratti linguistici provinciali ${ }^{9}$. Questi tratti erano di regola più arcaici di quelli cittadini, come spesso accade: infatti l'asse di variazione linguistica centro-periferia vede una distribuzione di forme più innovanti nei centri e più conservative nelle società più ristrette, specie se rurali. È frequente incontrare nelle parlate ebraiche degli arcaismi che non si sa se ascrivere alla segregazione o ai movimenti migratori dalla provincia alla città: i due fattori infatti hanno in questi casi lo stesso risultato.

L'esempio più noto, invece, di tratti interregionali, cioè provenienti da altre regioni italiane, sono i meridionalismi riscontrati sia nelle trascrizioni di "parlato" dialettale, sia nei testi più antichi di giudeo-italiano scritto ${ }^{10}$. Gli esempi di tratti provenienti da altre regioni sono numerosi: il dialetto giudeo-pitiglianese ha una base romana, in quanto era parlato da una comunità composta in larga parte di ebrei provenienti da Castro, città del viterbese distrutta dal Papa nel sec. XVI, e prima ancora da Roma. Elementi romani si trovano anche nel giudeo-livornese (Midgali 1990). A Modena appaiono affinità linguistiche con Mantova che possono essere interpretate come risultato di migrazioni:

"Questa analogia con Mantova non ci deve far concludere in favore dell'esistenza di una koiné nei due centri, ma è più semplicemente spiegabile con la frequenza di spostamenti e di contatti tra i nuclei ebraici dell'una città e dell'altra" (Massariello, 1977:48).

Un esempio chiaro questo della particolarità delle parlate giudeo-italiane: una mobilità geografica non solo diversa, ma maggiore, rispetto al resto della popolazione, mette la variante giudaica del dialetto al di fuori dei normali sviluppi storico-linguistici, che di solito mantengono differenze tra due città nella popolazione cristiana stabile.

\subsection{MISTILINGUISMO ESTERNO}

Si tratta della caratteristica forse più evidente di queste parlate e quella che non solo si è conservata fino a oggi, ma che è ancora produttiva: l'immissione di prestiti da lingue straniere. La fonte più rappresentata è l'ebraico, ma nelle varie comunità

9 Terracini spiega, per esempio, come monferrine alcune forme antiquate che riscontra per Torino (1938) e lo stesso avviene per il giudeo-ferrarese, dove si ritrovano voci provenienti da Lugo e da Cento (Massariello Merzagora, 1977:42): l'arcaismo in questo caso è il risultato del mistilinguismo.

10 L'esempio più frequentemente citato di influenza interregionale è quella meridionale, accertata per Roma (si veda peres. Scazzocchio 1970), ridimensionata da alcuni autori (Cuomo 1981) e recisamente negata per alcuni dialetti giudeo-italiani del Nord (Colomi 1971 per il mantovano e Fortis e Zolli 1979 per il veneziano). 
sono entrati anche prestiti dal tedesco, dallo spagnolo e dal portoghese, portati dai transfughi delle persecuzioni in quei Paesi.

\subsubsection{Prestiti da altre lingue europee}

Gli esempi di prestiti dallo spagnolo e dal portoghese sono molti, soprattutto per quelle comunità che accolsero molti ebrei iberici cacciati dalla Spagna e dal Portogallo nell'ultimo decennio del sec. XV. La comunità livornese, per esempio, fu fondata dagli esuli iberici, il giudeo-spagnolo e il giudeo-portoghese furono lingue vive per secoli (il primo come lingua orale, il secondo anche come la lingua scritta della comunità) e la parlata locale che si sviluppò, chiamata bagito, era piena di iberismi, come appare ancora dalle composizioni poetiche di Bedarida. Alcuni termini di derivazione iberica valicarono le mura delle comunità dove vivevano molti ebrei sefarditi, di origine iberica, e divennero comuni in altre parlate ebraiche, come negro, che non ha nessuna connotazione razziale, ma significa 'cattivo', 'brutto', 'negativo', come per l'italiano nero in espressioni come una giornata nera, una sfortuna nera.

I prestiti germanici sono meno numerosi, ma sono documentati, per esempio, sia per il giudeo-veneziano (Fortis e Zolli 1979) che per il giudeo-piemontese: proprio su questo punto nel $1893 \mathrm{G}$. Sacerdote apriva la nota su Il Vessillo Israelitico che costituisce l'inizio della presa di coscienza dell'esistenza delle parlate giudeo-italiane.

\subsubsection{Prestiti dall'ebraico}

I prestiti dalla lingua ebraica sono molti e presenti in ogni varietà di giudeo-italiano (e non italiano) di ogni area ed epoca, sia parlato che scritto: sono ancora oggi un tratto distintivo più o meno marcato dell'italiano parlato dagli ebrei.

I prestiti venivano a volte italianizzati, ma un largo numero sono rimasti intatti, sia pur con un adattamento dal sistema fonologico dell'ebraico a quelli dei singoli dialetti o dell' italiano sovraregionale (si veda per questo Artom 1962). In generale si è osservato che le alterazioni delle unità lessicali ebraiche sono scarse, minori di quanto non siano le alterazioni che subiscono generalmente $i$ prestiti da lingue straniere. Ciò potrebbe essere attribuito alla familiarità data dalla presenza dell'ebraico nella cultura e nel rito, anche se esso smise di essere usato come lingua parlata in epoca romana.

I prestiti non sono sempre gli stessi in tutte le varietà, ma coincidono in larga misura. La diffusione degli stessi prestiti in comunità diverse potrebbe essere dovuta agli spostamenti di famiglie e nuclei di cui abbiamo parlato, al valore universale di essi (termini ovvi del culto e dei realia ebraici) e, come suggerisce Massariello Merzagora (1977: 73), al fatto che i rabbini, che conoscevano e studiavano l'ebraico e che si spostavano da una comunità all'altra, potevano esserne i portatori.

La ragione più ovvia dei prestiti è quella di riferirsi a concetti e oggetti relativi al culto, alle festività e a realia della vita ebraica, come, per esempio, al cibo. Le ragioni 
in questo caso sono di tipo culturale e pratico. L'uso, a volte anche scherzoso, di espressioni del culto ricorda il ruolo del latino a livello popolare tra i cristiani, il cosiddetto 'latinorum', anch'esso, come l'uso dell'ebraico, sia serio che umoristico.

Abbiamo però anche ragioni di tipo psicologico per questi prestiti: l'uso di termini ebraici a scopo gergale e per ragioni eufemistiche. Il ricorso a termini stranieri come copertura dei termini proibiti, è un classico del procedimento eufemistico ${ }^{11}$. I termini colpiti da proibizione vengono investiti spesso violentemente di valenze negative a livello profondo, che risalgono all'infanzia. Per un procedimento psicologico naturale e molto forte, per esempio, la paura della morte e delle malattie mortali viene trasferita alle parole che le indicano, lo stesso avviene per la vergogna del sesso e della vita fisiologica, che in età infantile viene trasmessa alle parole $\mathrm{e}$, in epoche più religiose della nostra, per il timore di Dio (o di potenze magiche come la sfortuna e i suoi simboli). Il latino è stato ed è tuttora per l'italiano (e non solo per l'italiano) il serbatoio classico dei termini eufemistici. Il francese lo è stato nel secolo scorso e nella prima parte di questo secolo. È naturale che l'ebraico si sia prestato a questa funzione. Di questa origine sono, per esempio ${ }^{12}$ : ber ahaim dall'ebr. bet hahayim, lett. 'casa

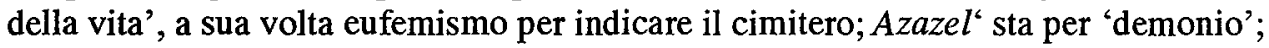
satan per 'diavolo'; ain ara per 'malocchio'; zona per 'prostituta'; sosanimme per 'seni'; musciau per 'gabinetto'; avel' per 'lutto' e 'persona in lutto'; teena per 'genitale femminile' e mila per 'membro virile'.

Un'altra ragione psicologica che ha portato alla proliferazione dei prestiti dall'ebraico è il loro uso gergale. Vi sono diversi modi di definire i gerghi. Un gergo è una parlata condivisa da un sottogruppo di parlanti all'interno di una comunità linguistica, che condividono una condizione particolare. La condizione condivisa può essere di natura diversa: abbiamo gerghi legati a un mestiere che rende necessaria tutta una serie di termini specifici, e in questo caso si parlerà, per esempio, di gerghi scientifici o di mestiere; oppure il gergo può servire a difendere il gruppo da attacchi esterni ed essere perciò un gergo segreto, come per la malavita o i partigiani e gli Alleati nella seconda guerra mondiale che usavano parole d'ordine e comunicavano in un codice cifrato; infine, il grupo può condividere una solidarietà più o meno scherzosa, come nei gerghi che si formano tra i giovani, o tra gli studenti o tra i soldati: in quest'ultimo caso lo scopo non è quello di nascondersi, ma di dare un segno di appartenenze al gruppo di identità.

Per gli ebrei, la funzione della lingua ebraica è forse stata nel passato anche quella di una lingua segreta, ma, in epoche recenti, l'uso gergale più produttivo è stato quello del linguaggio d'identità di gruppo e di gergo scherzoso. Tale è la natura dell'uso di molte delle parole, dei soprannomi scherzosi, dei proverbi, dei modi di dire di origine ebraica (ed anche non necessariamente solo di origine ebraica) che sono pecu-

11 Peri procedimenti eufemistici in generale e l'uso delle lingue straniere e di cultura a scopo di copertura dei termini proibiti, si veda Galli de' Paratesi N. (1964). 
liari dei dialetti giudeo-italiani. È questa una delle ragioni principali del fascino delle poesie di Bedarida e Del Monte e del teatro ebraico.

La vivacità delle formazioni gergali ebraiche è stata tale che dalle varietà giudeoitaliane, alcuni termini ebraici sono entrati nei dialetti locali: dal bagito degli ebrei di Livorno pare che in passato fossero passati dei termini al livornese dei gentili (Fornaciari 1983), lo stesso si era verificato per i dialetti del Nord e diversi termini del ghetto romano figurano in Belli e nei versi in romanesco di Luigi Zanazzo (Pavoncello 1978). Primo Levi nel suo libro Il sistema periodico parla di un gergo, con molte parole di giudeo-italiano, usato dai commercianti di stoffa, di cui esiste anche una versione ferrarese che è stata raccolta e che circola in forma solo dattiloscritta.

L'italiano nazionale è debitore all'ebraico di una parola solidamente affermata a livello nazionale, fasullo, per 'falso', 'non autentico' e di sciammannato, di diffusione romana e toscana, col signicato di 'sciatto, disordinato'.

\section{DEFINIZIONE, ORIGINI E RAPPORTI DELLE PARLATE EBRAICHE ITALIANE}

Dalle prime prese di coscienza dell'esistenza di parlate ebraiche dialettali tra la fine dell'Ottocento e l'inizio del Novecento ad oggi, si è assistito ad un fiorire di studi, soprattutto intorno ai documenti scritti religiosi del passato, oltre che naturalmente a quelli che Terracini aveva chiamato i residui delle parlate dialettali scomparse ormai definitivamente (con la sola eccezione del giudeo-romano). La pubblicazione dei documenti scritti si è intensificata negli ultimi anni e permette di avere oggi uno sguardo d'insieme nuovo sul problema.

In sostanza le domande che ci si sono poste nella letteratura sull'argomento possono essere così formulate:

1. Esisteva una koiné scritta di giudeo-italiano che stava ai diversi dialetti locali come l'italiano letterario stava alle diverse forme di volgare?

2. Se sì, quale era? Cioè di che origine regionale era?

3. Se esisteva una koiné scritta di giudeo-italiano, in che misura era anche parlata? Si può parlare di un "giudeo-italiano illustre"? Da una risposta positiva a questa domanda deriva che esso potrebbe avere influenzato le diverse forme dialettali locali.

\subsection{TEORIA DELLA KOINÉ GIUDEO-ITALIANA}

La prima di queste questioni è entrata d'impeto nella letteratura proprio nel momento in cui cominciavano gli studi dei testi scritti in giudeo-italiano. Infatti essa fu affrontata da Cassuto ${ }^{13}$ in uno dei suoi primi saggi sull'argomento: nel 1929 pubblica

13 Già prima di Cassuto altri studiosi avevano assunto implicitamente che esistesse un dialetto parlato dagli ebrei italiani piuttosto che delle versioni parzialmente diverse delle parlate locali: per esempio, Cologna nel 1829 afferma: "Noi abbiamo tra le mani un Breviario in rito italico in antico vernacolo 
l'edizione critica di un testo di una poesia religiosa in giudeo-italiano che fa risalire al sec. XIII, l'Elegia del 9 di Av, e qui presenta per la prima volta ${ }^{14}$ la sua tesi linguistica. Il punto saliente della tesi di Cassuto è che i MSS in giudeo-italiano che lui esamina hanno, come quello pubblicato nell'articolo, una patina centro meridionale:

“Tutte le traduzioni più antiche [di testi religiosi dall'ebraico in italiano] e i più antichi glossari ci presentano dialetti assai vicini tra loro, da classificarsi come dialetti centro-meridionali, e in particolare da assegnarsi alla sezione marchigiano-umbro-romanesca; vi prevalgono per lo più i caratteri dell'antico romanesco, non senza però qualche congruenza con altri dialetti della stessa sezione e anche con dialetti delle sezioni più meridonali." (375)

Su questa base, tenendo presenti gli eventi storici che investirono l'ebraismo italiano, cioè la cacciata dal Regno Spagnolo del Sud e le conseguenti migrazioni dal meridione a Roma, 'centro principalissimo dell' ebraismo', Cassuto postula:

“...una specie di Koiné ebraica, in cui prevaleva l'elemento romanesco, ma erano rappresentati anche elementi di altre provincie dello Stato della Chiesa e dell'Italia meridionale." (375

Le tendenze diacroniche che lo studioso mette in luce puntano ad un processo di convergenza, di standardizzazione verso la koiné, almeno a livello scritto:

"I testi più antichi ci presentano ancora divergenze dialettali abbastanza notevoli tra l'uno e l'altro, per il maggior rilievo che in ogni singola provincia vengono ad avere gli elementi locali, ma poi le differenze vanno attenuandosi". (376)

Non solo questa koiné creerebbe un polo di standardizzazione scritta attraverso l'imitazione del modello linguistico del corpus costituito dai testi religiosi tradotti, ma dal livello scritto si riverserebbe anche a livello orale, costituendo un riferimento anche per l'evoluzione delle lingue parlate dagli ebrei in tutta le penisola, anche al Nord:

"E poiché al nord di Roma le comunità di ebrei italiani... si costituirono in gran parte con elementi provenienti da Roma, la koiné giudaica a fondo romanesco si diffuse anche verso il nord in territori dialettali toscani, gallo-italici e veneziani, formando la base delle parlate giudaiche delle singole comunità, modificandosi via via più o meno rapidamente e più o meno profondamente sotto I'influsso dei dialetti locali...anche nei dialetti parlati restarono a lungo saldi molti elementi dell'originaria koiné centro-meridionale, e in parte essi persistono anche oggi in ciò che tuttora rimane dei dialetti giudaici: la grammatica del giudeo-fiorentino, ed esempio, coincide anche oggi in parte con quella del romanesco antico." (376)

Queste argomentazioni verranno riprese e ripetute in diversi scritti di Cassuto ${ }^{15}$ negli anni a venire, in pratica senza sostanziali cambiamenti, anzi in alcuni di questi scritti assumeranno formulazioni ancora più recise, data la sede in cui appariranno, come l'Encyclopædia Judaica (1932), dove la teoria è sintetizzata schematicamente in poche parole, o assume una forma divulgativa e quindi semplificata, come in 1930a.

\footnotetext{
ebreo-italiano [corsivo mio]..."

14 Si vedano 1930a, 1930b, 1930c,1932,1934.

15 Si vedano 1930a, 1930b, 1930c,1932,1934.
} 
$\mathrm{Su}$ questi argomenti Cassuto si riproponeva di tornare in modo organico, cosa che fu resa impossibile dagli eventi drammatici della guerra e della persecuzione. La sua teoria ebbe fortuna e fu ripresa forse in modo ancor più schematico da molti degli studiosi che seguirono, con poche eccezioni. Non è possibile qui ripercorrere l'uso che ne fu fatto dai suoi seguaci ${ }^{16}$ : tra di essi abbiamo anche Spitzer $(1942,1961)$. Non mancarono però coloro che dissentivano come fanno, più cautamente, la Fiorentino $\left(1937\right.$ e 1951-52) ${ }^{17}$ e, in modo reciso, Berenblut (1949) e, più tardi, Freedman (1972). Tanto Berenblut che Freedman possono essere portati come esempi tipici di oppositori alla teoria di Cassuto: ambedue sono molto recisi nel rifiutare la possibilità di una koiné. La loro critica è radicale, quello che intravedono è un caos dialettale, destrutturato e impossibile da descrivere, neppure in termini di tracce sistematiche anche contrastanti. Ambedue questi autori, che offrono un contributo valido come filologi dei testi, rifiutano di offrire per la parte linguistica una pars construens accanto a quella distruttiva. Berenblut in particolare, vede solo una polimorfia dialettale dei testi legata alla provenienza dialettale dei singoli estensori, appena mitigata dal ripetersi di formule fisse tradizionali di traduzione ${ }^{18}$.

16 Tra le rassegne critiche della letteratura quella che rende in modo completo e dettagliato ogni intervento è Freedman (1972, Cap.I) dove si potrà seguire la fortuna della teoria della koiné.

17 “Under such conditions we can consider Judeo-Italian as 'un veritable Yiddisch italien' [parole di Cassuto] only by a first glance, since Yiddish is a real linguistic entity isolated amidst alien languages and has its own characteristics very remote from those of the Judeo-Italian dialects" (1951-2: 77). L'infuenza dello Yiddish e del giudeo-spagnolo nel postulare un giudeo-italia no è stata in effetti molto forte. Cosí come è stata fondamentale l'influenza esercitata dal giudeo-francese e dal giudeo-romanzo. Ambedue questi termini furono usati da Blondheim, che Cassuto conosceva e stimava (si veda Blonheim 1931), e ripresi, senza le cautele critiche dell'originale, da una letteratura contro cui si scaglia Bannit (1963) che definisce senz'altro il giudeo-francese 'une langue fantôme'. La parabola della nascita, fortuna e confutazione del giudeo-francese come lingua a sé stante è in effetti parallela a quella del concetto di koiné giudeo-italiana.

18 La questione delle modalità fisse di traduzione dei testi biblici e rituali è molto interessante in sé e si interseca strettamente con la questione linguistica che stiamo trattando, perché il ripetersi di formule può essere dovuto a volte non al formarsi di una koiné ebraica scritta, ma al tramandarsi di formule usate solo in connessione con certi testi o tipi di testi. Quasi tutti gli autori che studiano i testi giudeo-italiani se ne sono occupati, da Cassuto (1929a, 1930c) in poi (si vedano Blondheim 2925 , Berenblut 1949: 242-251 e passim, Fiorentino 1951). Lo scopo di queste traduzioni dall'ebraico (e di quelle in latino prima $e$ in tante altre lingue in vari momenti) era quello di rendere trasparente l'originale del testo sacro per chi non conosceva più l'ebraico o lo conosceva appena. Esse quindi fungono come un testo a fronte spesso senza una sua autonomia o ricerca di buona lingua, al contrario più fedele possibile all'originale al punto da costituire una serie di calchi lessicali e sintattici e di formule ripetitive. Schwabb (1888: 285) descrive questo tipo di traduzione: "Diese Bedingung kann nur eine Übersetzung erfüllen, die nicht satzweise, sondern nur wortweise überträgt, die also die Wortstellung nicht ändert, die in Stil, Grammatik, ja so gar in Etymologie dem hebr. so gleichzukommen sucht, daß sie mehr hebr. fremdsprachlich ist: ja, daß sie ohne Original oft unverständlich bleibt". In realtà ognuno di noi ha prodotto simili traduzioni negli appunti fatti a scuola per poter ricordare il significato dei testi latini e greci da leggere. Freedman (1972: 103) usa addirittura il termine translationese. Sermoneta (1976:10, n. 19) fornisce un bell'esempio di questa forma mentis particolare, in cui mostra non solo che si passa dall'italiano all'ebraico traducendo alla lettera e non a senso, ma che avviene anche il contrario e che si ricalcano frasi italiane in ebraico. Il tradurre a 
Sarà però Terracini che affronterà per primo il materiale sia scritto che orale trascritto, con la doppia preparazione di glottologo e in particolare italianista e conoscitore dei problemi di ebraistica. Il primo intervento è del 1938, sulla scia dell'interesse antiquario e consiste nella pubblicazione di due poesie tradizionali in dialetto di Moncalvo commentate linguisticamente. Il secondo intervento nel 1957 è una lunga recensione-saggio al lavoro di Berenblut e il terzo la pubblicazione di materiale antiquario di varia origine nel $1962^{19}$.

La posizione di Terracini nei confronti della querelle sulla koiné è di equilibrata equidistanza e, in sostanza, le sue posizioni costituiscono ancora l'ossatura dell' interpretazione attuale, che è stata solo arricchita e collocata in una teoria esplicita sociolinguistica a partire dagli anni settanta.

Le proposte interpretative di Terracini partono dalla critica che egli fa di Berenblut che accusa di essere troppo reciso e schematico nelle sue posizioni. Da una parte è vero secondo Terracini che, come sostiene Berenblut, non è dimostrabile (e ancor meno lo era allora quando i testi pubblicati erano meno numerosi di oggi) se esistesse una koiné parlata né una vera e propria koiné scritta. Tuttavia da questo non si può passare a sostenere che $i$ testi esaminati abbiano ciascuno solo un carattere dialettale idiosincratico. Infatti, il terreno che sta fra una koiné scritta e l'uso di un dialetto interamente locale, è molto vasto e vi sono varie posizioni intermedie.

Il quadro che Berenblut si prospetta è, secondo Terracini, troppo radicalizzato, tra un'entità concepita troppo rigidamente, la lingua letteraria da una parte, e una serie di entità, i dialetti regionali concepiti in modo altrettanto irrealistico, dall'altra:

“[Berenblut parla]...come se l'italiano del ' 500 [epoca dei testi da lui esaminati] fosse stato quello che è oggi e non piuttosto una lingua il cui uso unitario era più che altro un ideale letterario ed estetico, espressione riservata ai più alti strati della cultura, non ancora scesa a penetrare di sé tutta la lingua viva ed estranea, quindi alla gran massa degli Ebrei per i quali l'italiano a maggior ragione rimaneva "volgare" e dialetta le... In realtà questo concetto rigido della lingua italiana circola in tutto quanto il volume (nel quale tutt'al più si ricorre ad una distinzione vaga e del tutto inadeguata come quella di 'italiano antico') e si accompagna ad un'idea altrettanto rigida e statica delle partizioni dialettali" (251-2).

Partendo da un'idea entificata e rigida di italiano letterario, da una parte (e non piuttosto di modello astratto o corpus scritto di riferimento in sé variegato) e dall'immagine ad essa speculare dei dialetti come sistemi a loro volta stagni (l'antitesi al concetto moderno del continuum linguistico), si finisce col crearsi, come quasi sempre nelle dispute scientifiche, un adversus quem in parte artificiale, una interpretazione schematica e semplificata della teoria del Cassuto sulla koiné scritta, a cui Cassuto, secondo Terracini, avrebbe solo accennato come tendenza. Partendo alla ricerca della

calco, è: “....abitudine scolastica, appresa durante l'infanzia ...sì che il testo stesso, tradotto con questo metodo, acquistava un valore e una patina di sacralità, essendone parimenti garantita la serietà scientifica. E il metodo di tradurre a calco diveniva addirittura un habitus mentale che accompagnava l'ebreo per tutta la vita" (1978: 68).

19 Sui contributi di Terracini si veda Segre 1968. 
koiné scritta e avendo in mente, come altra polarità possibile, dei dialetti completamente separati l'uno dall'altro, si finisce con passare dal primo estremo al secondo. La tinta dialettale c'è, ma, secondo Terracini:

"È una tinta dialettale diffusa più che esattamente determinata, una specie di conato di lingua letteraria che si profilava in questa scarna letteratura di scuola, il cui crogiuolo e centro di diffusione era Roma. Ciò è sicuro, e ne dobbiamo la precisa dimostrazione al Cassuto... Questo sfondo comune si alimentava poi di apporti regionali secondo la patria, o meglio la provenienza e residenza, dei singoli redattori" (244-5).

A questo "conato di lingua letteraria" limitata a una "scarna letteratura di scuola", si sovrapporrà più tardi, nel XVI sec., l'influenza della tendenza standardizzatrice verso il modello di lingua offerto dal corpus letterario italiano di impronta toscana:

... chiaro è ... il conato per svincolarsi dai ceppi dell'antica tradizone, chiarissima l'aspirazione ad avvicinarsi a quel tipo comune di lingua letteraria che incominciava allora a profilarsi in Italia, aspirazione che qualche anno più tardi diverrà proposito nel famoso glossario biblico di Leone da Modena" (p.245).

Queste due tendenze di convergenza verso modelli di lingua scritta, quello centro-meridionale ebraico prima e quello toscano nazionale poi, "conati" diversi di standardizzazione verso una forma di lingua sovraregionale non necessariamente allargata al parlato, coesisteranno nei diversi autori coll'inerzia dei sistemi di provenienza di ciascuno di essi, che costituiranno l'elemento base della mistura. Questa mistura porterà Berenblut a concludere, quasi con disperazione:

'The impression gathered from the preceding survey of the language of our MSS is one of utter confusion. We find ourselves faced with a muddle of contradictory phenomena and seemingly irreconciable influence. Not only do we encounter opposing data in different MSS, but even in one and the same..." (p.197).

Si tratta del fenomeno ben noto dell'oscillazione di chi si muove tra sistema proprio e sistema di riferimento, o comunque di interferenza, fenomeno che il sociolinguista considera centrale nei suoi dati e non caotico, ma coerente e in effetti sistematico, tanto da essere descrivibile, nella sociolinguistica moderna, come tendenza statistica, mediante l'uso delle regole variabili.

Per Terracini sono chiare le tracce meridionali e romanesche nel dialetto di Ferrara, come la marcata resistenza alla riduzione e dileguo delle vocali atone: tracce, anche se ormai pallidissime, molto scarsamente documentate e quindi poco analizzabili, non da intendersi come resti di una vera e propria koinè, ma piuttosto, di mistilinguismo esterno portato sulla scorta degli spostamenti verso Nord (per Ferrarra va ricordato il dominio papale a partire dal sec. XVI). In modo analogo si possono spiegare tracce meridionali nel giudeo-pitiglianese (gli ebrei di Pitigliano provenivano da Roma attraverso Montalto di Castro), nel giudeo-livornese (Midgali Della Torre 1990) e nel giudeo-fiorentino e le unità lessicali, per la verità rare (Modena Mayer e Massariello Merzagora 1973: 936-937), presenti nei dialetti del Nord: Colorni (1971) interpreta queste forme come veri e propri prestiti, esempi di mistilinguismo esterno 
piuttosto che prove di un'antica koiné centro-meridionale espansa al Nord. L'interpretazione di Terracini e il rifiuto di Colorni di accettare la koinè per i dialetti giudeo-italiani del Nord vengono ripresi e articolati da Jochnowitz (1972).

Negli ultimi quindici anni abbiamo un largo numero di traduzioni di testi giudeoitaliani. Il materiale a disposizione quindi si è notevolmente ampliato. Inoltre, nelle discussioni sull'argomento si riflettono i cambiamenti avvenuti nella linguistica, dove ormai l'impostazione di fondo della ricerca è permeata dagli studi di sociolinguistica.

Con l'avvento del modello di Labov, cioè l'introduzione dei parametri extralinguistici a cui può essere correlata rigorosamente la variazione, l'uso delle tecniche statistiche della sociolinguistica quantitativa e l'introduzione delle regole variabili nel modello generativo dove vanno a convivere con le regole categoriche, abbiamo ormai gli strumenti per misurare la variabilità che prima era o trascurata da parte di molti studiosi in quanto variazione libera o casuale, o avvertita anche con molta finezza (per l'argomento che stiamo trattando è il caso di Terracini) ma non descritta e misurata, perché mancavano ancora gli strumenti tecnici. Rimanendo all'interno del sistema linguistico stesso, ancorati alle regole categoriche della linguistica strutturale e chomskiana, prima dell'introduzione dei parametri extralinguistici, delle tecniche statistiche e delle regole variabili, non si poteva scorgere il valore sistematico e non casuale delle distribuzioni di tratti in variazione e variabilità e sistematicità venivano viste come due fatti contradditori.

Negli ultimi anni le posizioni degli studiosi sono molto più sfumate e articolate dal punto di vista sociolinguistico: Sermoneta, che era partito dall'accettazione della teoria di Cassuto di una koiné scritta e parlata (1963 e 1971), se ne allontana di fatto e introduce, in un un lungo articolo pubblicato in due parti (1976 e 1978), una definizione senz'altro molto più sfumata, che è, in sostanza, una riformulazione, basata su un'analisi puntuale di vari testi, della posizione di Terracini e non molto lontana dall'accettazione senza riserve di quest'ultima che si trova in Jochnowitz (1974). Quello che caratterizzerebbe, secondo Sermoneta, le varietà di questi testi non sarebbe una koiné che li accomuna tutti, ma un modo linguistico (8) dovuto ad un atteggiamento linguistico $(17)^{20}$ particolare degli ebrei d'Italia, che avrebbe prodotto caratteristiche peculiari dei testi, come l'arcaicità e una fluidità particolare rispetto alle date situazioni linguistiche locali. Poiché per fluidità si intende quello che si indica tecnicamente con variabilità, quello che Sermoneta sostiene qui è che le varietà giudeo-italiane hanno una loro variabilità diversa e aggiunta a quella delle varietà italiane non giudaiche, quindi una doppia variabilità, quella interna alle varietà di italiano e quella propria, rispetto a queste ultime.

20 In realtà il termine atteggiamento linguistico proviene da L. Cuomo, come lei stessa dichiara (1981: 436, n. 32). Va ricordato però che il termine in linguistica è comunemente usato ad indicare qualcosa di diverso. Esso traduce linguistic attitude e si riferisce alla valutazione linguistica positiva e negativa, cioè la percezione della propria varietà e di quelle degli altri, in termini di lealtà linguistica, accettazione o rifiuto, fino alle opinioni stereotipanti. 
Per quanto riguarda l'atteggiamento linguistico è un'espressione che suggerisce la coscienza da parte del parlante delle proprie scelte linguistiche, che è possibile solo in parte (per esempio per la scelta di vocaboli ebraici o ebraicizzanti e per l'abitudine alla traduzione a calco), ma che non è postulabile per una larga parte dell'uso fatto dagli ebrei dei sistemi o sottosistemi linguistici, dovuto alla loro storia. Per esempio, nel caso dell'oscillazione tra sistema nativo e sistema di prestigio ebraico romano-meridionale nei testi giudeo-italiani, oppure tra sistema locale usato anche dai gentili e sistema precipuo usato solo dagli ebrei nell'uso linguistico all'interno delle comunità, è più esatto, come per tutti i parlanti che oscillano tra due o più sistemi, descrivere la variabilità linguistica come una serie di scelte in larga parte inconscie ai parlanti, condizionate dal loro appartenere ad un gruppo sociale particolare. Il comportamento che sta dietro la variabilità linguistica, di oscillazione tra sistemi o sottosistemi in lizza, è il risultato di forze che sono al di sotto della soglia della decisione conscia. Tant'è vero che molti parlanti scelgono e credono di avere un determinato accento per ragioni di prestigio e di immagine, ma in realtà oscillano tra quello che vorrebbero avere e quello che hanno di fatto, senza avere il pieno controllo del proprio comportamento linguistico. È per questo che si preferisce parlare in sociolinguistica di condizione sociolinguistica o sociale, come fattore che determina le scelte.

La studiosa che recentemente ba mediato in modo brillante la sua specialità di esegeta e filologa di studi ebraici con un'approfondimento teorico-linguistico è $\mathrm{L}$. Cuomo, che, rifacendosi all'impostazione data da Terracini, la articola con gli strumenti analitici della sociolinguistica della variazione (1981 e 1982). Benché mantenga il termine giudeo-italiano, dà ad esso il valore di "varietà linguistiche specifiche di un gruppo sociologico", non di una lingua o dialetto unitario. L'autrice è anche conscia della complessità delle tensioni centrifughe e centripete all'interno della società italiana durante tutto il periodo in cui furono stesi i testi nelle varietà giudeo-italiane, delle tendenze alla formazione di koiné regionali, della dicotomia parlato-scritto e tiene conto di tutte le dimensioni di variazione che possono razionalizzare la descrizione dell'eterogeneità linguistica di questi documenti e mostrarne la sistematicità.

\section{CONCLUSIONI}

Il modo nuovo di concepire l'universo dei dati linguistici, ormai fatto proprio anche dagli studiosi di questa tematica, permette di mettere a punto una definizione delle varietà giudeo-italiane sia scritte che orali.

La rivoluzione operata dalla sociolinguistica non ha solo permesso di ristudiare in modo più approfondito realtà linguistiche, invero molto rare, di relativa omogeneità, per esempio lingue altamente standardizzate come inglese o francese, ma ha soprattutto fornito un modello per comprendere realtà non standardizzate, come quella che abbiamo esaminato, dove l'estrema variabilità spingeva le interpretazioni in due direzioni opposte e polarizzate. Da una parte, si rischiava di entificare una lingua fantasma unitaria, la koinè giudeo-italiana, dall'altra si tendeva a liquidare l'universo 
dei dati linguistici, come quelli del giudeo-italiano, come un caos dialettale dove regnava la variazione casuale (come facevano Berenblut e Freedman).

Tale variazione può invece essere messa a fuoco in un quadro teorico se la riconsideriamo alla luce della nuova concezione dell'universo dei dati linguistici come vengono concepiti nella macrosociolinguistica moderna. Nell'analisi sincronica questo approccio teorico permette la misurazione della variabilità non più come 'libera' o 'casuale', ma come sistematica e perciò ordinata. Addirittura dati empirici di questo tipo possono essere letti al di là della sincronia, in termini di tendenza diacronica, come io stessa ho cercato di fare per l'italiano standard (1984), dove la particolare distribuzione delle varianti standard e non, vista in termini di previsioni di tendenza può essere misurata statisticamente. Nel caso delle varietà di giudeo-italiano, invece, trattandosi di varietà estinte (escluso forse il caso di Roma), il modello di Labov non può più essere usato a livello di misurazione empirica, ma può fornire comunque un quadro di riferimento e una struttura esplicativa potente, su cui situare a livello di ipotesi teorica articolata l'universo dei dati variabili che ci sono giunti.

Le varietà giudaico-italiane scritte presentano chiaramente fenomeni di tendeza e di variabilità, risultati di forze diverse, attrazioni verso varietà che fungono da modello e reazioni verso un sistema portante di fondo, quello dell'estensore. Nulla vieta di ipotizzare che quest'ultimo potesse contemporaneamente subire l'influenza dei modelli di traduzione letterale di cui s'è parlato, con i calchi dall'ebraico e formule físse e anche una tendenza standardizzatrice verso il modello di lingua scritta centro-meridionale diffusa dalla Roma ebraica.

Queste due tendenze diverse potevano coesistere e dover mediare le scelte linguistiche che ne risultavano col sistema di fondo della parlata del nostro estensore ipotetico, se era diverso: la tendenza standardizzatrice in questo caso esercitando una forza antitetica a quella del sistema nativo. Sulle scelte dell'estensore, poi, possono essersi sommate quelle del copista o dei copisti, a loro volta posti di fronte, come sempre tutti i parlanti, a più di un sistema in lizza. Come minimo vi è la scelta tra sistema nativo e quello del modello letterario, quando i due non coincidano. Questa differenza si elimina, o piuttosto diventa una differenza solo di registro quando vi è una standardizzazione molto avanzata, sia scritta che orale, cioè per l'Italia solamente in epoca recente e non per tutti i parlanti.

La provenienza diversa delle forme che appaiono nei testi è frutto di questa variabilità e quello che può essere definito come peculiare dei testi stessi e della lingua in essi usata, come nota Jochnowitz (1974: 214), non è necessariamente il singolo tratto, che si può anche ritrovare in testi non ebraici, ma l'insieme delle co-occorrenze di tratti, ciascuno dei quali, preso separatamente, può essere comune anche con altre parlate. Quello che è precipuo non è il singolo tratto (e la polemica sul singolo tratto ${ }^{21}$

21 Molta dell'energia dei ricercatori che si sono occupati dei documenti di giudeo-italiano è stata dedicata in gran dettaglio allo studio dei singoli tratti (quelle che Freedman ha chiamato painstakingly written monographs), nella presunzione che essi isolatamente dessero indicazioni che permettessero di 
polverizza il concetto di tendenza), ma la co-occorrenza di fasci di tratti, ciasscuno dei quali può non essere precipuo se preso isolatamente. I fasci di co-occorrenze poi formeranno non necessariamente un sistema, ma caratteristiche con occorrenze tendenziali non prevedibili sulla base di regole categoriche ma descrivibili in teoria con regole variabili.

Definire le parlate giudeo-italiane significa tentare di descriverle a due livelli: all'esterno e all'interno. La definizione esterna significa descriverle rispetto alle varietà di italiano contigue nel tempo, nello spazio e sul parametro sociale e di registro. Descriverle all'interno significa mettere in luce la variabilità precipua che esse presentano.

\subsection{DEFINIZIONE DELLE VARIETÀ GIUDEO-ITALIANE COME VARIETÀ ETNICHE}

Abbiamo visto che le varietà giudeo-italiane sono state spesso anche indicate come dei "dialetti". Nel contesto italiano il termine dialetto indica dei sistemi regionali diversi dalla lingua nazionale o standard ${ }^{22}$, situati lungo un continuo rispetto a quest'ultima. Ad un estremo di questo continuo linguistico abbiamo l'italiano e all'altro abbiamo parlate che non possono essere descritte come sottosistemi dell' italiano, ma come qualcosa di diverso, che in realtà potremmo considerare in certi casi lingue diverse. Ad esse riserviamo il termine dialetto invece di lingua per ragioni extralinguistiche: esse infatti non hanno lo status sociale di ufficialità che si associa al termine lingua.

È evidente che le parlate giudeo-italiane non possono essere dialetti in quanto non sono sistemi diversi dall'italiano legati ad una variazione geografica. Si può, in effetti, usare il termine dialetti giudeo-italiani, solo intendendo però le varianti giudaiche dei singoli dialetti italiani. Per esempio, il dialetto giudeo-piemontese è la variante ebraica del dialetto piemontese, ma non un dialetto con sistema a sé stante rispetto all'italiano e al piemontese.

In nessun caso abbiamo sistemi completamente diversi dall'italiano, dalle sue varietà o dai dialetti, parlati dagli ebrei (come accade per lo yiddish e per il giudeospagnolo in Turchia e Bosnia), tanto meno poi sull'asse di variazione geografica,

definire geograficamente la varietà in questione. Per esempio, quasi tutti gli autori (Berenblut 1949: 201;Terracini1951:63, n.1; 1957: 254; 1962: 267, n.11; Bedarida 1956: XVII; Scazzocchio 1970: 114; Hijmans-Tromp 1989: 233) considerano la desinenza plur. femm. $-i$ come un indicatore di influenza meridionale. Si tratta in realtà di un tratto fuorviante se considerato in isolamento, come nota la Cuomo: “...il fenomeno del plurale femminile di prima declinazione in -i è largamente diffuso,...in area centro-meridionale, toscana e settentrionale (1982: 26)". Secondo Terracini (1957: 254) era "ben marcato in certe parti della Toscana, per es. nell'antico lucchese...verso Nord e verso il centro si diffuse, probabilmente come segno dell'irradiare toscano..." ben lungi da essere un segno marcato inequivocabile di influenza meridionale.

22 Nella linguistica anglosassone invece si parla non solo di dialetti regionali ma anche di dialetti sociali, per indicare le varietà diastratiche o socioletti. 
come varianti regionali, né sull'asse periferica, come varianti urbane rispetto a quelle rurali o viceversa.

Neppure il parametro sociale ci aiuta a definire le parlate giudeo-italiane, perché gli ebrei non sono definibili come una classe sociale e queste varietà non sono socioletti. Esse presentano in realtà una loro stratificazione sociale interna, anche se probabilmente leggermente diversa da quella dei socioletti dell'italiano, in quanto gli ebrei come comunità linguistica hanno avuto e hanno tuttora una stratificazione sociale diversa in parte da quella del resto della popolazione.

Per definire lo status di queste varietà all'interno dei sottosistemi dell'italiano, sarebbe utile forse un'analogia con lo status linguistico del Black English: in tutti e due i casi la differenziazione linguistica è funzione di una differenza etnica che è legata a condizioni sociali diverse e comporta una cultura diversa. Si propone quindi di definire queste varietà varietà etniche o etnico-culturali all'interno delle parlate di italiano.

\subsection{RAPPORTI TRA LE VARIETÀ ETNICHE GIUDEO-ITALIANE}

Le particolari condizioni degli ebrei rispetto alla società circostante e le peculiarità della loro cultura condizioneranno il loro comportamento linguistico lasciando tracce nella fisionomia e nell'evoluzione delle loro parlate.

Abbiamo già discusso altrove nel testo (par. 3) le caratteristiche linguistiche che differenziano le parlate giudeo-italiane dalle altre varietà di italiano e i fattori extralingustici che determinano queste differenze. Vorremmo soffermarci su un aspetto diacronico: le diverse spinte che le parlate ebraiche banno subito nel contesto della standardizzazione dell'italiano.

Per processo di standardizzazione si intende l'effetto di attrazione e sovrapposizione che una determinata varietà esercita sulle altre, se e quando viene dotata di particolare prestigio sociale, politico o culturale. Questo crea un movimento centripeto in favore della varietà di prestigio che entra in conflitto, sia con le forze centripete di coesione dei singoli sistemi, sia con altre possibili tendenze standardizzatrici in atto. Le altre tendenze standardizzatrici possono essere di due tipi: locali, a livello geografico più ristretto, oppure forze alternative allo stesso livello sovraregionale, a raggio più o meno vasto. Vorrei suggerire qui il modello del cono che ho già usato altrove per descrivere questo processo (1984: 47). Se si immagina di circoscrivere la carta geografica dell'Italia nella base di un cono, il cui apice è su Firenze, il cono ci rende la sovrapposizione dell'italiano di Firenze sulle varietà del resto del paese, che giocano il ruolo di sostrato, e il cerchio alla base visualizza il concetto di forza centripeta esercitato dalla varietà di prestigio. Ma per ciascuna regione, all'interno del cono che ha come apice la varietà dotata di prestigio sovraregionale, dobbiamo immaginare a sua volta dei coni regionali, ai cui apici si trovano le varietà maggiormente dotate di prestigio locale, spesso quelle dei capoluoghi, che sono bacino di immigrazione e di scambi (asse di variazione centro-periferia). Sotto questi coni se ne possono immagi- 
nare altri minori, sempre su altri assi centro-periferia, che ne possono sottendere altri ancora minori e così via all'infinito.

Per la cultura ebraica dobbiamo immaginare che, oltre a questa stratificazione vera e propria di coni che visualizzano tensioni centripete e controtensioni centrifughe nei confronti di varietà dotate di prestigio o importanza via via minore, vi fosse un cono alternativo a livello di lingua scritta sovraregionale: quello sotteso dalla varietà centro-meridionale che veniva usata a Roma. La differenza di cultura creava infatti, all'interno delle comunità ebraiche, dei riferimenti di prestigio linguistico diversi e in più rispetto a quelli della cultura italiana. Abbiamo visto che la Roma ebraica, sede della maggiore comunità del Paese e centro di studi illustre, ebbe, nel Medioevo, un'influenza culturale paragonabile a quella della Firenze di Dante, Petrarca e Boccaccio per il resto del Paese (dell'influenza culturale di Firenze risentì naturalmente anche la minoranza ebraica, che non rimase mai, anche nei momenti peggiori, completamente isolata dalla cultura circostante). Il prestigio culturale della varietà centromeridionale parlata a Roma fu rafforzato dalle migrazioni di ebrei meridionali da Roma a Ferrara, Pitigliano, Firenze e Livorno.e altrove.

$\grave{E}$ chiaro quindi adesso il quadro teorico con cui si possono in teoria definire le varianti che si presentano nei testi: sono in gioco varianti diatopiche, cioè quelle locali contro quelle sovraregionali provenienti dalle spinte di due tendenze standardizzatrici, quella solo ebraica centro-meridionale e quella nazionale toscana; sono sempre diatopiche, ma di origine diversa, le varianti di mistilinguismo interno portate fisicamente dalle migrazioni, incluse quelle sull'asse centro-periferia in epoca moderna, con l'urbanesimo susseguente alla scomparsa dei ghetti dei piccoli centri; abbiamo varianti diacroniche tra testo e testo legate ai diversi momenti in cui $i$ documenti furono redatti: bisogna ricordare che i riferimenti linguistici diacronici per le varietà giudeo-italiane sono diversi da quelli del resto delle varietà italiane, perché l'isolamento introduce conservatismo e perciò arcaismi; abbiamo varianti di registro innanzi tutto tra parlato e scritto e anche, all'interno dei documenti scritti, tra testo e testo, a seconda del livello di formalità e letterarietà a cui i diversi testi si pongono, date le diverse destinazioni funzionali e di pubblico che essi avevano. Ai due estremi abbiamo forse l'Elegia del 9 di Av da una parte e i manuali di preghiere dall'altra ${ }^{23} \mathrm{e}$, in mezzo, tutta una gamma di testi la cui differenziazione risulta interpretabile se viene posta su un parametro che è insieme funzionale e di registro (ma anche diastratico dato il diverso livello educativo dei destinatari dei diversi testi) in cui le diverse destinazioni di pubblico e il tipo di materia trattata dettavano livelli diversi di formalità, dialettalità vs. letterarietà e proporzioni diverse di letteralità nella traduzione dall'ebraico.

Accanto alle varianti sociolinguistiche, abbiamo altre di altra natura, legate non a variabilità di sistema, ma all'uso. Sono le varianti lessicali e sintattiche, legate alla

23 Si veda a questo proprosito Cuomo (1981: 440-441). L'una è un pregevole testo poetico, gli altri sono traduzioni pedisseque concepite per i semi-letterati, donne e bambini. 
particolare tradizione delle traduzioni bibliche, che porta a formule, calchi e modalità di traduzione, che costituiscono quasi una vera e propria lingua settoriale, specifica del corpus della letteratura giudeo-italiana.

Infine non dobbiamo dimenticare che abbiamo delle varianti legate alla stratificazione filologica: sono quelle introdotte dai copisti sui particolari idioletti già compositi dei traduttori o autori dei testi.

Lo stato di cose che stiamo descrivendo ricorda quello che è stato scritto per l'italiano dei secoli passati ed è valido ancora per l'italiano di oggi a livello orale, anche se a livello scritto adesso abbiamo uno standard nazionale di riferimento: si veda la discussione nel mio lavoro sulla standardizzazione dell'italiano (1984: 48, in particolare II. 3 "Dinamica dei rapporti tra standard e altre forme di lingua: forze centripete e forze centrifughe"). Da quando l'eterogeneità e la differenziazione possono essere descritte come strutturate in distribuzioni statistiche significative e sono quindi occorrenze chiare in termini sia matematici che esplicativi, vengono ormai viste come intrinseche e fisiologiche a tutti i sistemi linguistici.

È così che si possono vanificare molte delle "lingue fantasma", per usare il termine di Bannit, supposti sistemi unitari e discreti, che hanno popolato la glottologia prima e la linguistica strutturale poi, tra la fine del secolo scorso e questo secolo, come il giudeo-francese, il Vulgärlatein, il "francese popolare", lo "spagnolo popolare" e, in Italia, l"italiano popolare" che ancora si cita come un tipo di italiano a sé.

Anche l'italiano standard orale non è per il momento che un modello di prestigio astratto, al vertice di un cono, limpidamente e caratteristicamente strutturato in termini distributivi lungo i parametri classici, diastratico, diatopico e di registro (si veda, nel mio lavoro sulla standardizzazione (1984), in particolare II.6, "I risultati statistici"). Evocarlo come un sistema discreto in uso sarebbe un'entificazione impropria.

Proprio questa analogia, tra l'italiano orale "colto parlato dai fiorentini" e il giudeo-italiano, fa pensare che Cassuto nel postulare quest'ultimo, avesse come riferimento in qualche modo, come modello mentale inconscio, la storia dell'italiano, come se gli fosse venuto spontaneo di ipotizzare per gli autori della letteratura giudeo-italiana, un modello di riferimento, quello romano di origine centro-meridionale, con la stessa funzione storica e unificatrice che la varietà di Firenze ha avuto per l'italiano.

Non a caso, in apertura del mio Lingua toscana in bocca ambrosiana mettevo dei versi di Lewis Carroll da The Hunting of the Snarck, che in effetti richiamano il concetto del fantasma inafferrabile:

"You may seek it with thimbles - and seek it with care

You may hunt it with forks and hope;

You may threaten its life with a railway-share;

You may charm it with smiles and soap". 
"But oh, beamish nephew, beware of the day,

if your Snark be a Boojoum! For then

You will sofly and suddenly vanish away,

And never be met with again!".

Si potrebbe dire che, in tutti e due i casi, se si commette l'errore di valicare i limiti teorici e si va al di là di modelli astratti di prestigio normativo, di tendenze e co-occorrenze, e si entificano sistemi discreti, questi si vendicano trasformandosi in un Boojoum. Ho riconosciuto con immediata simpatia, 1" "effetto Boojoum" in alcune osservazioni di L. Cuomo (1982: 29), che era giunta per vie lontanissime dalle mie alla stessa sensazione, nel suo caso per il giudeo-italiano. Cuomo, infatti, parla del giudeo-italiano come di un Proteo:

"[Gold con il conio del termine Italkian] ...chiude la gabbia in cui si è creduto di intrappolare un Proteo: ma questo, in una delle sue innumerevoli trasformazioni, se ne sguscia fuori, lasciando la gabbia ben chiusa, ma vuota"

La similitudine dell'animale da catturare mi era stata anch'essa suggerita dall'italiano standard, quando, insieme ai versi di Lewis Carroll, sceglievo il seguente brano dantesco come intestazione in apertura al mio libro:

"Quam multis varietatibus latio dissonante vulgari, decentiorem atque illustrem Italiae venemur loquelam, et ut nostrae venationi pervium callem habere possimus, perplexos frutices atque sentes prius eiciamus de silva" (Dante Alighieri, De vulgari eloquentia, I.11.1),

dove la selva mi pareva descrivesse bene l'intricatezza dell'eterogeneità data dalle variabili, prima che l'introduzione dei moderni metodi analitici permettesse di mostrarne il disegno in effetti coerente e strutturato.

\section{INDICE DEI TESTI CITATI}

AGUS I.A., 1974, "The Languages Spoken by Askenazic Jews in the High Middle Ages", in Joshua Finkel Festschrift, New York: 19-28.

ARTOM E.S., 1962, “La pronuncia dell'ebraico presso gli Ebrei d'Italia” in La Rassegna di Israel - Scritti in memoria di Federico Luzzatti XXVII: 26-30

ASCOLI G.I., 1890, “Una lettera del Comm. G.I. Ascoli” in Il Vessillo Israelitico XXXVIII: 114.

BACHI R., 1926, "Ricerche folcloristiche e linguistiche degli Ebrei d'Italia" in Rassegna mensile di Israel II: 362-367

_ 1929 , "Saggio sul gergo di origine ebraica in uso presso gli Ebrei torinesi verso la fine del sec. XIX" in Rassegna mensile di Israel IV: 21-35

BANNITT M., 1963, "Une lange fantôme: le judéo-français" in Revue de Linguistique moderne XXVII 1963: 245-294

BECCANI A., 1941, "Saggio storico-linguistico sugli ebrei a Livorno", Bollettino Storico Livornese V: 269-277. 
$\longrightarrow, 1942$, "Contributo alla conoscenza del dialetto degli Ebrei di Livorno" in Italia dialettale 18: 189-202

BEDARIDA G., 1956, Ebrei di Livorno. Tradizioni e gergo in 180 sonetti giudaico livornesi, Firenze.

BENE'KEDEM, 1936, “La Gnora Luna', scene di vita ebraico-fiorentina” in Rivista Mensile d'Israel 6: 547-580, ristampa Firenze, 1977.

BERENBLUT M., 1949, A Comparative Study of Judeo-Italian translations of Isaiah, New York.

BIRNBAUM S.A., 1942, “Jewish Languages", in EPSTEIN I., LEVINE E. e ROTH C. (a c. di) Essays in Honour of the Very Rev. Dr. J.H. Hertz, Londra.

_ 1971, "Jewish Languages", in Enclyopoedia Judaica, Gerusalemme, 10: 66-69.

BLONDHEIM D.S., 1923, “Essai d'un vocabulaire comparatif des parlers romans des Juifs au Moyen Age", in Romania, 4: 343-388; 526-569.

$\ldots, 1925$, Les parlers judéo-romains et la Vetus latina. Etude sur les rapports entre les traductions bibliques en langue romane des Juifs au moyen-âge et les anciennes versions. Parigi.

- 1931 , Recensione a U. Cassuto Travaux sur le judéo-italian in Romania, 57: 440-443.

BONFIL R., 1991, Gli Ebrei in Italia nell'epoca del Rinascimento, Firenze.

CAMERINI D., 1909, "Ancora qualche osservazione sugli studi dialettali" in Vessillo Israelitico, LVII: 505-507.

CAMMEO D., 1909, "Studi dialettali" in Il Vessillo Israelitico LVII: 169-170, 214215, 314-315, 359-361, 459-461, 504-505.

—, 1910, "Studi dialettali" in Il Vessillo Israelitico LVIII 8-9, 148-149, 403-404, 448-450, 506-507, 543-545.

_., 1911, "Studi dialettali" in Il Vessillo Israelitico LIX: 25-26, 52-53, 102-104, 143-144.

CASSUTO U., 1909, "Parlata ebraica" in Vessillo Israelitico: 254-260

_ 1926 , "La vetus latina e le traduzioni giudaiche medioevali della Bibbia" in Studi e materiali di Storia delle religioni, Roma, II:145-62.

— 1929, “Un' antichissima elegia in dialetto giudeo-italiano" in Silloge linguistica dedicata alla memoria di Graziadio Isaia Ascoli Torino: 349-408.

___, 1930a, "Il libro di Amos in traduzione giudeo-italiana" in Miscellanea in memoria di H.P.Chajes, Firenze: $19-38$

,$- 1930 b$, "La Teffillah delle nostre nonne" in Rivista Mensile d'Israel: 144-48

1930c, "La tradizione giudeo-italiana per la traduzione della Bibbia" in Atti del Primo Congresso Nazionale delle Tradizioni Popolari Firenze: 114-21.

_ 1930 d, "Les traductions judéo-italiennes du rituel" in Revue des Études Juives LXXXIX 1930: 260-280. 
_ 1932, "Jüdisch-italienisch" in Encyclopaedia Judaica Berlino IX: 555.

_ 1934-5, "Saggi delle antiche traduzioni giudeo-italiane della Bibbia" in Annuario di Studi ebraici del Collegio Rabbinico Italiano, Roma, I: 101-34

COLOGNA A., 1829, Prefazione a LUZZATO S.D. Formulario delle orazioni secondo il rito italiano, Vienna.

COLOMBO D., 1970, "Il ghetto di Moncalvo e una sua poesia" in La Rassegna Mensile di Israel, XXXVI: 436-441

COLORNI V., 1971, "La parlata degli ebrei mantovani" in Scritti in memoria di $A$. Milano. La Rassegna Mensile di Israel, XXXVII: 109-164

CUOMO L., 1981, "Il giudeo-italiano e le vicende linguistiche degli ebrei d'Italia" in Italia giudaica. Atti del I Congresso Internazionale, Bari, 18-22 Maggio: 427454

_. 1982, "Italiano vs. giudeo-italiano, vs. 0 (zero): una questione metodologica" in Italia, III: 7-32.

- 1988, Una traduzione giudeo-romanesca del libro di Giona, Tübingen.

DEL MONTE C., 1908, Sonetti giudaico-romaneschi, Roma.

_ 1927, Sonetti giudaico-romaneschi, Firenze.

— 1932, Nuovi sonetti giudaico-romaneschi, Roma.

—, 1955, Sonetti postumi giudaico-romaneschi e romaneschi, Roma.

EPSTEIN I., LEVINE E. e ROTH C. (a c. di), 1942, Essays in Honour of the Very Rev. Dr. J.H. Hertz, Londra.

FIORENTINO G., 1937, “Note lessicali al Maqré Dardaqé. Archivio Glottologico italiano, 29: 138-60.

—, 1951-2, "The General Problems of Judeo-Romance in the Light of the Maqré Dardaqé, in The Jewish Quarterly Review, N.S., 17: 57-77, ristampa dell'opuscolo Il Maqré Dardaqé e alcune questioni sullo studio della parlata giudeo-roman$z a$, Torino 1938.

FORNACIARI P.E., 1983, “Aspetti dell'uso del 'Bagitto' da parte dei gentili”. Rassegna Mensile d'Israel, XLIX: 432-454

FORTIS U., 1989, Il ghetto in scena. Teatro giudeo-italiano nel Novecento. Storia e testi, Roma.

FORTIS U., ZOLLI P., 1979, La parlata giudeo-veneziana, Roma.

FREEDMAN A., 1972, Italian Texts in Hebrew Characters: Problems of Interpretation, Wiesbaden

GALLI DE' PARATESI N., 1964, Semantica dell'eufemismo. L'eufemismo e l'interdizione linguistica nell'italiano contemporaneo, Torino.

$\longrightarrow, 1984$, Lingua toscana in bocca ambrosiana Milano.

GOLD J.L, 1980, “The Glottonym Italian” in Italia, II: 98-102. 
HIJMANS-TROMP I., 1989, Mosé da Rieti, Filosofia Naturale e fatti de Dio. Testo inedito del sec. $X V$, Leida

JOCHNOWITZ G., 1972, "Forme meridionali nei dialetti degli ebrei dell'Italia centrale" in Rassegna mensile di Israel, XXXVIII: 424-429

$\ldots, 1974 a$, "Parole di origine romanza ed ebraica in giudeo-italiano" Rassegna mensile di Israel, 212-219.

$\longrightarrow, 1974 b$, Recensione a Freedman Italian Texts in Hebrew Characters: Problems of Interpretation, in Romance Philology XXVIII: 213-217.

LEVI P., 1975, Il sistema periodico, Torino.

MASSARIELLO MERZAGORA G., 1977, Giudeo-italiano. Dialetti italiani parlati dagli Ebrei d'Italia, Pisa.

— 1980, "Le parlate giudeo-italiane" in Studi, Fatti, Ricerche, 12: 12-14

MIDGALI (DELLA TORRE) M., 1990, "Residui della parlata giudaico-romanesca nel giudaico livornese" in Italia, IX: 115-126

MILANO A., 1963a, Storia degli Ebrei italiani, Milano.

—_, 1963b, Il ghetto di Roma, Roma

MODENA MAYER M., 1978, "Osservazioni sul tabù linguistico in giudeo-livornese in Saggi in memoria di U. Nahon, a cura di R. Bonfil, D. Carpi, M. Modena Mayer, G. Romano, G.B. Sermoneta, Gerusalemme: 166-179.

MODENA MAYER M., MASSARIELLO MERZAGORA G., 1973, "Il giudeo-modenese negli appunti R. Giacomelli" in Rendiconti dell'Istituto Lombardo di Scienze e Lettere, CVII: 863-938.

MODONA L., 1893, "Intorno ad un possibile lavoro filologico sui dialetti parlati già dagli ebrei d'Italia" in Vessillo Israelitico, XLI: 60-62, 85-88, 121-123, 154-157.

PAVONCELLO N., 1978, "Gli ebrei nell'opera di Luigi (Giggi) Zanazzo" L'urbe, 4: 15-21

— 1986, Modi di dire ed espressioni dialettali degli ebrei di Roma, Roma.

— 1988, Modi di dire ed espressioni dialettali degli ebrei di Roma, Roma.

POLACCO B., 1972, "Quarant'anni fa. Tre tempi in giudeo-veneziano" in Rassegna Mensile d'Israel, 38: 584-617

SACERDOTE G., 1893, "Di alcune voci dialettali e corrotte tra gli Israeliti piemontesi" Vessillo israelitico XLI: 14-17.

SCHWAB M., 1888, "Le maqré Dardaqé", in Revue des Études Juives, 16: 253-268; 17: 111-124, 285-298.

SEGRE C., 1968, "Benvenuto Terracini, linguista, e le parlate giudeo-italiane" in Rassegna mensile di Israel 34: 327-333

SERMONETA G., 1963, "Una trascrizione in caratteri ebraici di alcuni brani filosofici della Commedia" in Romanica et Occidentalia, études dédiées à la mémoire de Hiram Peri, Oxford: 23-42 
—_, 1971, "Judeo-Italian" in Encyclopædia Judaica Gerusalemme 10: 427-29.

— 1976 , "Considerazioni frammentarie sul giudeo-italiano" in Italia 1 (1) 1976: $1-29$.

_ 1978 , "Considerazioni frammentarie sul giudeo-italiano II. Testi italiani in caratteri ebraici" in Italia 1 (2) 1978: 62-106.

SESTIERI SCAZZOCCHIO L., 1970, "Sulla parlata giudaico-romanesca" in Scritti in Memoria di Enzo Sereni Gerusalemme: 101-132

SPITZER L., 1942, "Judeo-Italian" in The Universal Encyclopedia New York VI: 255-56.

- 1961, "The influence of Hebrew and Vernacular Poetry on the Judeo-Italien Elegy, in Twelfth Century Europe and the Foundations of Modern Society a c. di M. Clagett, Madison 1961: 115-130.

STOCK M., 1970, "Una poesia d'occasione in dialetto ebraico-triestino" in Rassegna mensile di Israel 36: 366-368

TERRACINI B., 1938, "Due composizioni in versi giudeo-piemontesi del secolo XIX" in Rassegna mensile di Israel XII: 164-183

_. 1951, "Residui di parlate giudeo-italiane raccolte a Pitigliano, Roma, Ferrara" in Rassegna mensile di Israel XVII: 3-11, 63-72, 113-121

- 1957, Recensione di M. Berenblut A Comparative Study of Judeo-Italian translations of Isaiah," in Romance Philology, X: 243-258.

_ 1962 , "Le parlate giudaico-italiane negli appunti di Raffaele Giacomelli" in Scritti in Memoria di F. Luzzato, Rassegna mensile di Israel XXVII: 260-295

WEINREICH M., 1956, "The Jewish Languages of Romance Stock and Their Relation to Earliest Yiddish", in Romance Philology, 9: 402-428.

ZOLLI P., 1979, La parlata giudeo-veneziana, Roma

Povzetek

JUDOVSKA ITALIJANŠČINA IN PROBLEMI DEFINICIJE.

POGLAVJE IZ ZGODOVINE JEZIKOSLOVJA

\footnotetext{
Študija načenja vprašanje t.i. judovske italijanščine. S tem izrazom razumemo jezik, v katerem so judovske skupnosti v Italiji pisale med XI in XVIII stoletjem, dokazana pa je tudi raba v živem govoru, ponekod celo do začetka našega stoletja.

$\mathrm{V}$ prispevku se tehta teorija U. Cassuta iz tridesetih let, po kateri naj bi imela judovska koiné svoje temelje v južni in srednji Italiji, kar je pač bilo v duhu historičnega in strukturalnega gledanja tistega časa. V luči modernega sociolingvističnega gledanja ne gre toliko za jezikovne kot za etnično-kulturne variante.
} 